\title{
Fat-soluble vitamins from diet in relation to risk of type 2 diabetes mellitus in Japanese population
}

\author{
Ehab S. Eshak ${ }^{1,2 *}$, Hiroyasu Iso ${ }^{1}$, Isao Muraki ${ }^{1}$ and Akiko Tamakoshi ${ }^{3}$ \\ ${ }^{1}$ Public Health, Department of Social Medicine, Graduate School of Medicine, Osaka University, Osaka 565-0871, Japan \\ ${ }^{2}$ Public Health and Community Medicine Department, Faculty of Medicine, Minia University, 61511 Minia, Egypt \\ ${ }^{3}$ Department of Public Health, Faculty of Medicine, Hokkaido University, Sapporo 060-8638, Japan
}

(Submitted 14 August 2018 - Final revision received 3 December 2018 - Accepted 9 December 2018 - First published online 31 January 2019)

\section{Abstract}

The role of fat-soluble vitamins in the pathology of type 2 diabetes needs further research. Possible protective effects could be expected for vitamins $\mathrm{A}$ and $\mathrm{E}$ via their antioxidant properties, vitamin $\mathrm{K}$ via its modulating effects on cytokines and insulin resistance and vitamin $\mathrm{D}$ via the enhancement of insulin sensitivity. However, the evidence on association between fat-soluble vitamins from diet and risk of diabetes is limited. Therefore, among 19168 healthy Japanese of both sexes aged 40-79 years, we used the logistic regression analyses to examine the prospective association between FFQ-estimated dietary fat-soluble vitamins (A, K, E and D) and the risk of type 2 diabetes incident over a 5-year period. During this 5-year period, 494 new cases of diabetes were self-reported. Vitamins K and E from diet were associated with lowered risk of incident diabetes, whereas no associations with dietary intake of vitamin A or D were observed. The multivariable OR in the highest $v$. lowest quartiles of intakes were $0.71\left(95 \% \mathrm{CI} 0.54,0.93, P_{\text {trend }}=0.01\right)$ for vitamin $\mathrm{K}$ and $0.72(95 \% \mathrm{CI} 0.55,0.95, P$ trend $=0.02)$ for vitamin E. Mutual adjustment for dietary intake of these vitamins did not change the association. There were no interactions with sex, age, smoking status, BMI or having a family history of diabetes, $P$ were $>0 \cdot 10$. In conclusion, higher dietary intake of fat-soluble vitamins $\mathrm{K}$ and $\mathrm{E}$, but not vitamin $\mathrm{A}$ or $\mathrm{D}$, were associated with lowered risk of type 2 diabetes among Japanese population.

Key words: Fat-soluble vitamins: Vitamin A: Vitamin K: Vitamin E: Vitamin D: Diabetes: Japanese

Type 2 diabetes is a chronic disease with multiple factors involved in its pathogenesis and/or consequences ${ }^{(1)}$. In the past decade, there was a notable increase in diabetes prevalence in Japan $^{(2)}$. Environmental determinants including reducing diet quality play a role in type 2 diabetes burden ${ }^{(3,4)}$

The main pathology in type 2 diabetes is linked to the metabolism of energy and macronutrients. However, recent studies have shown that micronutrients are also involved ${ }^{(4,5)}$. It has been found that fat-soluble vitamins with antioxidant effects, such as vitamins A and E, may have protective effects against oxidative stress-driven pathogenesis and complications of chronic diseases including type 2 diabetes ${ }^{(5-9)}$. Moreover, interests have also been shown ${ }^{(5,10-14)}$ in the possible roles of the other two fat-soluble vitamins, vitamins $\mathrm{K}$ and $\mathrm{D}$.

However, so far, most of the available evidence is limited to either comparing the plasma levels of these fat-soluble vitamins among healthy individuals and patients with diabetes or the effect of supplementation with some of these vitamins on risk of diabetes $^{(5,6)}$. The evidence for associations between dietary intakes of these vitamins and the risk of developing diabetes is scarce $^{(6-14)}$.
The reduced risk of diabetes was reported with higher $\beta$-carotene and $\alpha$-tocopherol dietary intake in Swedish men ${ }^{(6)}$; whereas in Dutch ${ }^{(7)}$ and Finnish ${ }^{(8)}$ populations, dietary intake of carotenoids other than $\beta$-cryptoxanthin were not associated with reduced risk. However, dietary intake of vitamin $\mathrm{E}$ was not associated with the risk of type 2 diabetes in a Chinese study ${ }^{(9)}$.

A reduced risk of type 2 diabetes was observed with increased intake of vitamin $\mathrm{K}$ in a prospective Spanish ${ }^{(10)}$ and Dutch studies ${ }^{(11)}$. However, phylloquinone supplementation was not associated with reduced risk in a conclusion of a systematic review ${ }^{(12)}$.

Growing body of evidence suggests an important role of vitamin $\mathrm{D}$ in risk modification of diabetes ${ }^{(13-16)}$. However, the association of dietary vitamin $\mathrm{D}$ and risk of type 2 diabetes is inconsistent according to the available studies ${ }^{(13,14)}$. Dietary vitamin $\mathrm{D}$ intake was inversely associated with the metabolic syndrome in the Women's Health Study ${ }^{(13)}$ but not with risk of incident diabetes in the EPIC-InterAct study ${ }^{(14)}$.

In the present analysis, we aimed to test our hypothesis that higher dietary consumption of fat-soluble vitamins (A, K, E and D) could be inversely associated with the risk of incident type 2 diabetes among a large cohort of Japanese population. 


\section{Methodology \\ Study population}

Among residents of forty-five Japanese communities who participated in the Japan Collaborative Cohort Study for Evaluation of Cancer Risk (JACC Study), that was launched in 1988-1990, the final study subjects were 19168 healthy Japanese men and women aged 40-79 years, without missing information for both dietary intake of fat-soluble vitamins and history of diabetes at two time points, the baseline and 5 years later (see online Supplementary Fig. SI). Those participants completed a selfadministered questionnaire about their medical histories, diet and other habits after. The detailed protocol for the JACC study was described elsewhere ${ }^{(17)}$. Participants or community leaders on their behalf have given informed consents, and the ethics committees of Osaka and Hokkaido Universities have approved the protocol of this investigation.

\section{Dietary assessment}

Participants completed their past year habitual consumptions of forty food and drink items in a FFQ that allowed five possible choices of consumption's frequencies: rarely, 1-2 times/month, 1-2 times/week, 3-4 times/week to almost every day without specifying portion size ${ }^{(18)}$. The daily intake of each item in the FFQ was calculated by multiplying these frequencies by $0,0.38$, $1.5,3.5$ and $7 \cdot 0 /$ week, respectively. With the aid of Japanese food composition tables, fat-soluble vitamin content from each item was specified and multiplied by the subject's consumption frequency score of this item. Summing the total values for the forty items of the FFQ gave the daily dietary intakes of fatsoluble vitamins. The portion size was specified from a validation study among eighty-five participants of the JACC study who completed $3 \mathrm{~d}$ of weighted dietary records over 1 year period consumptions ${ }^{(18)}$. The FFQ-estimated intake of fatsoluble vitamins were moderately correlated with those estimated from dietary records of the validation study as indicated by the Spearman rank correlation coefficients $(0.37$ for vitamin A, 0.38 for vitamin E, 0.39 for vitamin $\mathrm{D}$ and 0.40 for vitamin K) as shown previously ${ }^{(19)}$.

\section{Assessment of diabetic status}

The 5-year cumulative incidence of diabetes was used because the precise date of diabetes diagnosis was unknown. Individuals with incident diabetes were baseline diabetes free individuals who reported, on the 5-year survey, being diagnosed by physicians to have diabetes. This self-reported diagnosis of diabetes among our participants was validated by comparison with therapy data and laboratory findings and had 70 and $95 \%$ sensitivity and specificity in men and 75 and $98 \%$, in women, respectively. Details for criteria used to diagnose diabetes were given previously ${ }^{(4)}$.

\section{Statistical analysis}

We categorised dietary intake of fat-soluble vitamins into quartiles and tested for differences in individuals' baseline characteristics across the increasing intake quartiles using ANCOVA and $\chi^{2}$ test. Using the lowest quartiles of intakes as a reference, logistic regression analysis was used to estimate the OR and $95 \%$ CI of incident type 2 diabetes in the other three quartiles of intake for vitamins $\mathrm{A}, \mathrm{K}, \mathrm{E}$ and $\mathrm{D}$. With the large sample size of the present study, the multivariable model was adjusted for age, sex, non-dietary factors including family history of diabetes (yes, no), past history of hypertension (yes, no), smoking status (never, former smoker, current smoker of 1-19 and $\geq 20$ cigarettes/d), BMI (quartiles), walking hours (almost no, daily $0.5,0.6-0.9$ and $\geq 1 \mathrm{~h}$ ), exercise hours (almost no, weekly 1-2 , 3-4 and $\geq 5 \mathrm{~h}$ ) and supplement use of vitamin E, vitamin $\mathrm{C}$ and multivitamins (yes, no). The final model was adjusted further for dietary factors including alcohol intake (never, former and current daily drinker of 0.1-22.9, 23.0-45.9, $46.0-68.9$ and $\geq 69.0 \mathrm{~g}$ ethanol), green tea intake in cups (<once/week, 1-6/week, 1-2/d, 3-5/d and $\geq 6 /$ d), coffee intake in cups (<once/week, 1-6/week, $1-2 / \mathrm{d}$ and $\geq 3 / \mathrm{d}$ ), total energy intake (quartiles) and energy-adjusted intake of $\mathrm{Mg}$, total dietary fibre and carbohydrate (quartiles). For each vitamin, we also considered the mutual adjustment for intake of three other fat-soluble vitamins in an additional model. The median dietary intake values in quartiles of fat-soluble vitamins were entered into the model as continuous variables and their significance values represented the $P_{\text {for trend }}$.

Because potential synergy between some of these vitamins regarding other health outcomes was suggested in a previous report $^{(20)}$, we investigated the combination effect of dietary intake of the studied vitamins below and above the median intake of our study population. We also ran several stratifying analyses by age and BMI median values ( 54 years and $25 \mathrm{~kg} / \mathrm{m}^{2}$ ), sex, smoking status, having a family history of diabetes. A cross-product term of the dietary intake of each fatsoluble vitamin and the dichotomous stratifying variables was conducted and tested for its $P$ value in the regression model. Our large sample size allowed the $80 \%$ statistical power for testing, and a significance level for a two-sided $P$ value $<0.05$ using the SAS 9.4 software (SAS Institute Inc.) was applied for all statistical analyses.

\section{Results}

Among 19168 Japanese men and women, male participants represented $36.9 \%$, the mean age was 55.7 (SD 9.5) years and the 5 -year cumulative incidence of diabetes was $2.6 \%$ in total (3.5\% among men and $2.0 \%$ among women). The main food sources that contributed to the dietary intake of fat-soluble vitamins in our data were liver (86.2\%) and fried vegetables (11.1\%) for vitamin $\mathrm{A}$; spinach or garland chrysanthemum $(81.1 \%)$ and potatoes $(15.1 \%)$ for vitamin $\mathrm{K}$; fresh meat (37.9\%), fresh fish $(27 \cdot 1 \%)$ and spinach (14.3\%) for vitamin E; and fresh fish (77.7\%) and fried vegetables (17.6\%) for vitamin $\mathrm{D}$ (not shown in tables).

Table 1 shows that individuals in the highest quartiles of fatsoluble vitamins intake compared with those in the lowest quartiles were older, less likely to smoke but were more likely to walk, to practice sports, and to drink green tea and were with less carbohydrate but higher Mg intakes. 
Table 1. Participants' baseline characteristics according to quartiles of fat-soluble vitamin intakes (Mean values and standard deviations; numbers and percentages)

\begin{tabular}{|c|c|c|c|c|c|c|c|c|c|c|c|c|c|c|c|c|c|c|}
\hline & \multicolumn{9}{|c|}{ Quartiles of vitamin A intake } & \multicolumn{9}{|c|}{ Quartiles of vitamin K intake } \\
\hline & \multicolumn{2}{|c|}{1 (low) } & \multicolumn{2}{|c|}{2} & \multicolumn{2}{|c|}{3} & \multicolumn{2}{|c|}{4 (high) } & \multirow[b]{2}{*}{$P_{\text {trend }^{*}}$} & \multicolumn{2}{|c|}{1 (low) } & \multicolumn{2}{|c|}{2} & \multicolumn{2}{|c|}{3} & \multicolumn{2}{|c|}{4 (high) } & \multirow[b]{2}{*}{$P_{\text {trend }^{*}}$} \\
\hline & Mean & SD & Mean & SD & Mean & SD & Mean & SD & & Mean & SD & Mean & SD & Mean & SD & Mean & SD & \\
\hline Subjects $(n)$ & \multicolumn{2}{|c|}{4792} & \multicolumn{2}{|c|}{4792} & \multicolumn{2}{|c|}{4792} & \multicolumn{2}{|c|}{4792} & & \multicolumn{2}{|c|}{4792} & \multicolumn{2}{|c|}{4792} & 47 & & 47 & & \\
\hline Age (years) $\dagger$ & $55 \cdot 6$ & 9.9 & 55.7 & $9 \cdot 6$ & 55.5 & 9.3 & $56 \cdot 0$ & $9 \cdot 1$ & 0.04 & $55 \cdot 2$ & 9.9 & $55 \cdot 0$ & $9 \cdot 6$ & 55.9 & 9.3 & $56 \cdot 7$ & 9.1 & $<0.001$ \\
\hline Males (\%) & 3 & & & & & & 3 & & 0.001 & 4 & & & & 3 & & 3 & & $<0.001$ \\
\hline Family history of diabetes (\%) & 8 & & & & & & 7 . & & 0.24 & 8. & & & & 9 & & 7 . & & 0.09 \\
\hline History of hypertension (\%) & & & & & & & 1 & & 0.003 & 1 & & & & 1 & & 1 & & 0.38 \\
\hline Current smoker (\%) & 2 & & & & & & 1 & & 0.02 & 2 & & & & 2 & & 1 & & 0.02 \\
\hline BMI $\left(\mathrm{kg} / \mathrm{m}^{2}\right)$ & $22 \cdot 7$ & 3.0 & $22 \cdot 7$ & $2 \cdot 8$ & 22.8 & 2.8 & 22.6 & $2 \cdot 7$ & 0.08 & $22 \cdot 7$ & 3.0 & $22 \cdot 6$ & $2 \cdot 8$ & $22 \cdot 8$ & 2.8 & $22 \cdot 8$ & 2.8 & 0.17 \\
\hline Sports $\geq 5 \mathrm{~h} /$ week $(\%)$ & & & & & & & 3 & & $<0.001$ & 2 & & 2 & & 2 & & 2 & & $<0.001$ \\
\hline Walking $\geq 5 \mathrm{~h} /$ week (\%) & & & & 3 & & & 5 & & $<0.001$ & 4 & & 5 & & 5 & & 6 & & $<0.001$ \\
\hline Ethanol intake $(\mathrm{g} / \mathrm{d})$ & 27 & 23 & 27 & 21 & 26 & 22 & 27 & 23 & 0.85 & 27 & 23 & 27 & 23 & 26 & 22 & 27 & 22 & 0.58 \\
\hline$\geq 1$ cup of green tea/d & 6 & & & 9 & & & 7 & & $<0.001$ & 6 & & 7 & & 7 & & 7 & & $<0.001$ \\
\hline$\geq 1$ cup of coffee/d & & & & & & & 3 & & 0.95 & 4 & & 3 & & 3 & & 3 & & $<0.001$ \\
\hline Energy intake (kJ/d) & 5828 & 1807 & 6376 & 1745 & 6803 & 1728 & 7422 & 1983 & $<0.001$ & 5690 & 1757 & 6402 & 1699 & 6766 & 1770 & 7573 & 1908 & $<0.001$ \\
\hline Carbohydrate intake $(\mathrm{g} / \mathrm{d})$ & 255 & 34 & 245 & 32 & 239 & 31 & 229 & 33 & $<0.001$ & 253 & 34 & 244 & 32 & 240 & 32 & 230 & 33 & $<0.001$ \\
\hline Mg intake (mg/d) & 195 & 36 & 220 & 37 & 242 & 35 & 256 & 43 & $<0.001$ & 187 & 33 & 219 & 32 & 238 & 32 & 269 & 35 & $<0.001$ \\
\hline Vitamin A intake $(\mu \mathrm{g} / \mathrm{d})$ & 500 & 125 & 802 & 70 & 1058 & 83 & 2079 & 1149 & $<0.001$ & 721 & 640 & 959 & 653 & 1200 & 760 & 1560 & 979 & $<0.001$ \\
\hline Vitamin K intake $(\mu \mathrm{g} / \mathrm{d})$ & 121 & 42 & 171 & 54 & 214 & 56 & 249 & 70 & $<0.001$ & 99 & 24 & 156 & 14 & 212 & 18 & 189 & 33 & $<0.001$ \\
\hline Vitamin E intake (mg/d) & $3 \cdot 6$ & $1 \cdot 2$ & 4.8 & 1.2 & 5.7 & 1.2 & 6.7 & 1.8 & $<0.001$ & $3 \cdot 3$ & 1.0 & 4.7 & 0.9 & $5 \cdot 6$ & 1.1 & $7 \cdot 1$ & 1.4 & $<0.001$ \\
\hline Vitamin D intake ( $\mu \mathrm{g} / \mathrm{d})$ & $6 \cdot 0$ & $3 \cdot 2$ & 7.5 & $3 \cdot 4$ & 8.6 & 3.5 & $9 \cdot 9$ & 3.9 & $<0.001$ & 5.5 & 3.0 & $7 \cdot 4$ & $3 \cdot 1$ & 8.5 & 3.4 & $10 \cdot 6$ & $3 \cdot 7$ & $<0.001$ \\
\hline & & & & Quartiles & of vitam & E intake & & & & & & & Quartiles & of vitamir & D intake & & & \\
\hline & 1( & & & & & & $4(\mathrm{hi}$ & gh) & & $1(1$ & & 2 & & 3 & & $4(\mathrm{~h}$ & gh) & \\
\hline & Mean & SD & Mean & SD & Mean & SD & Mean & $\mathrm{SD}$ & $P_{\text {trend }^{*}}$ & Mean & $\mathrm{SD}$ & Mean & SD & Mean & SD & Mean & SD & $P_{\text {trend }^{*}}$ \\
\hline Subjects $(n)$ & & & & & & & 475 & & & 47 & & 47 & & 47 & & 47 & & \\
\hline Age (years) $\dagger$ & 55.7 & $10 \cdot 1$ & 55.4 & 9.5 & 55.6 & $9 \cdot 3$ & $56 \cdot 2$ & 9.0 & 0.002 & 55.8 & $10 \cdot 1$ & 55.5 & 9.6 & 55.4 & $9 \cdot 3$ & $56 \cdot 1$ & 8.9 & 0.07 \\
\hline Males (\%) & & & & & & & 3 & & $<0.001$ & 3 & & 3 & & 3 & & 3 & & 0.18 \\
\hline Family history of diabetes (\%) & & & & & & & 7. & & 0.006 & 8. & & & & 7. & & 7 . & & 0.006 \\
\hline History of hypertension (\%) & 1 & & & & & & 15 & & 0.05 & 1 & & 1 & & 1 & & 1 & & 0.45 \\
\hline Current smoker (\%) & & & & 1 & & & 17 & & 0.01 & 2 & & 2 & & 2 & & 2 & & 0.03 \\
\hline $\mathrm{BMI}\left(\mathrm{kg} / \mathrm{m}^{2}\right)$ & $22 \cdot 7$ & 3.0 & $22 \cdot 7$ & $2 \cdot 8$ & 22.7 & $2 \cdot 8$ & 22.8 & $2 \cdot 8$ & 0.26 & $22 \cdot 7$ & $2 \cdot 9$ & $22 \cdot 7^{-}$ & $2 \cdot 8$ & $22 \cdot 7$ & $2 \cdot 8$ & 22.8 & 2.8 & 0.008 \\
\hline Sports $\geq 5$ h/week (\%) & 2 & & & & & & 2 & & $<0.001$ & 2 & & 2 & & 2 & & 2 & & $<0.001$ \\
\hline Walking $\geq 5 \mathrm{~h} /$ week (\%) & & & & 3 & & & $6 z$ & & $<0.001$ & 5 & & 5 & & 5 & & 6 & & $<0.001$ \\
\hline Ethanol intake $(\mathrm{g} / \mathrm{d})$ & 28 & 23 & 27 & 23 & 25 & 22 & 27 & 22 & 0.04 & 26 & 22 & 25 & 22 & 27 & 23 & 29 & 22 & $<0.001$ \\
\hline$\geq 1$ cup of green tea/d & & & & & & & 7 & & $<0.001$ & 6 & & 7 & & 7 & & 7 & & $<0.001$ \\
\hline$\geq 1$ cup of coffee/d & & & & & & & 3 & & $<0.001$ & 4 & & 3 & & 3 & & 3 & & $<0.001$ \\
\hline Energy intake $(\mathrm{kJ} / \mathrm{d})$ & 5489 & 1715 & 6255 & 1619 & 6745 & 1591 & 7937 & 1828 & $<0.001$ & 5586 & 1669 & 6280 & 1653 & 6828 & 1703 & 7736 & 1920 & $<0.001$ \\
\hline Carbohydrate intake $(\mathrm{g} / \mathrm{d})$ & 256 & 35 & 246 & 32 & 239 & 30 & 225 & 31 & $<0.001$ & 256 & 32 & 246 & 31 & 238 & 32 & 226 & 34 & $<0.001$ \\
\hline Mg intake (mg/d) & 186 & 33 & 217 & 30 & 240 & 30 & 270 & 35 & $<0.001$ & 199 & 42 & 223 & 37 & 235 & 37 & 258 & 40 & $<0.001$ \\
\hline Vitamin A intake $(\mu \mathrm{g} / \mathrm{d})$ & 688 & 544 & 997 & 666 & 1177 & 733 & 1598 & 1020 & $<0.001$ & 821 & 642 & 1044 & 740 & 1168 & 786 & 1406 & 1000 & $<0.001$ \\
\hline Vitamin K intake $(\mu \mathrm{g} / \mathrm{d})$ & 111 & 39 & 165 & 42 & 209 & 45 & 272 & 52 & $<0.001$ & 139 & 60 & 177 & 61 & 201 & 65 & 240 & 70 & $<0.001$ \\
\hline Vitamin E intake (mg/d) & $3 \cdot 1$ & 0.7 & 4.5 & 0.3 & 5.6 & 0.3 & 7.5 & 1.1 & $<0.001$ & 3.6 & 1.2 & 4.7 & 1.2 & 5.5 & 1.3 & 6.8 & 1.6 & $<0.001$ \\
\hline Vitamin D intake $(\mu \mathrm{g} / \mathrm{d})$ & 4.8 & $2 \cdot 3$ & $7 \cdot 0$ & $2 \cdot 7$ & 8.7 & 3.0 & 11.6 & 3.2 & $<0.001$ & 3.7 & 1.0 & $6 \cdot 2$ & 0.7 & 8.9 & 1.0 & $13 \cdot 3$ & 1.9 & $<0.001$ \\
\hline
\end{tabular}

* The $x^{2}$ test was used for categorical variables; ANOVA was used for continuous variables.

† Means and standard deviations (all such values). 
In minimally adjusted models that controlled for age and sex, increasing dietary intake of vitamins $\mathrm{K}$ and $\mathrm{E}$ were associated with lowered risk of incident type 2 diabetes, whereas no significant association with dietary intakes of vitamin A or D was observed (Table 2). Further adjustment for non-dietary and dietary factors did not change the associations materially; the multivariable $\mathrm{OR}$ in the highest $v$. the lowest quartiles of intakes was $0.71(95 \%$ CI $0.54,0.93, P$ trend $=0.01)$ for vitamin $\mathrm{K}, 0.72$ $(95 \%$ CI $0.55,0.95, P$ trend $=0.02)$ for vitamin E, $0.82(95 \% \mathrm{CI}$ $0.62,1.07, P_{\text {trend }}=0.15$ ) for vitamin A and 0.84 (95\% CI 0.63 , $\left.1 \cdot 11, P_{\text {trend }}=0 \cdot 19\right)$ for vitamin D. Mutual adjustment for dietary intakes of these vitamins did not change the association materially.

We could not prove any synergetic effects between the dietary intake of fat-soluble vitamins on the risk of diabetes as suggested by the analyses of combination categories of intake above and below the median intake of these vitamins (online Supplementary Tables SI and SII).
There were no significant interactions with sex, age, smoking status, BMI or having a family history of diabetes, $P>0 \cdot 10$ (data not shown in tables).

\section{Discussion}

The present study is the first to shed light on the possible association between dietary intake of fat-soluble vitamins and the risk of type 2 diabetes in a large cohort of Asians. Findings indicated that dietary intake of both vitamins $\mathrm{K}$ and $\mathrm{E}$ but not dietary intake of vitamin A or D were associated inversely with diabetes risk. These associations persisted even after controlling for traditional diabetes risk factors and other dietary variables and did not vary by different levels of a wide range of participants' characteristics.

The antioxidant properties of vitamins A and E were the main interest of the research that investigated the impact of dietary

Table 2. Associations between dietary intakes of fat-soluble vitamins and the risk of 5 -year incidence of type 2 diabetes (Odds ratios and $95 \%$ confidence intervals; numbers and percentages)

\begin{tabular}{|c|c|c|c|c|c|c|c|c|}
\hline & \multicolumn{7}{|c|}{ Quartiles of fat-soluble vitamin intakes } & \multirow[b]{3}{*}{$P_{\text {trend }}{ }^{*}$} \\
\hline & \multirow[b]{2}{*}{ Q1 (low) } & \multicolumn{2}{|c|}{ Q2 } & \multicolumn{2}{|c|}{ Q3 } & \multicolumn{2}{|c|}{ Q4 } & \\
\hline & & OR & $95 \% \mathrm{Cl}$ & OR & $95 \% \mathrm{Cl}$ & OR & $95 \% \mathrm{Cl}$ & \\
\hline Subjects $(n)$ & 4792 & & & & & & & \\
\hline \multicolumn{9}{|l|}{ Vitamin A } \\
\hline \multicolumn{9}{|l|}{ Cases } \\
\hline$n$ & 141 & & & & & & & \\
\hline$\%$ & 2.9 & & & & & & & \\
\hline Model $1 \dagger$ & 1.00 & 0.90 & $0.70,1 \cdot 15$ & 0.83 & $0.65,1.07$ & 0.79 & $0.65,1.07$ & 0.07 \\
\hline Model $2 \ddagger$ & 1.00 & 0.92 & $0.72,1.17$ & 0.85 & $0.66,1.10$ & 0.84 & $0.65,1.08$ & 0.16 \\
\hline Model 3§ & 1.00 & 0.91 & $0.71,1 \cdot 16$ & 0.85 & $0.65,1 \cdot 10$ & 0.82 & $0.62,1.07$ & 0.15 \\
\hline Model 4\| & 1.00 & 1.00 & $0.77,1.21$ & 0.93 & $0.70,1.21$ & 0.89 & $0.71,1.18$ & 0.29 \\
\hline \multirow{2}{*}{\multicolumn{9}{|c|}{$\begin{array}{l}\text { Vitamin K } \\
\text { Cases }\end{array}$}} \\
\hline \multicolumn{4}{|l|}{ Cases } & & & & & \\
\hline$n$ & 152 & & & & & & & \\
\hline$\%$ & 3.2 & & & & & & & \\
\hline Model $1 \dagger$ & 1.00 & 0.79 & $0.62,1.01$ & 0.77 & $0.60,0.99$ & 0.71 & $0.55,0.91$ & 0.009 \\
\hline Model 2‡ & 1.00 & 0.81 & $0.63,1.03$ & 0.78 & $0.61,1.01$ & 0.74 & $0.57,0.94$ & 0.02 \\
\hline Model $3 \S$ & 1.00 & 0.79 & $0.62,1.02$ & 0.77 & $0.59,0.99$ & 0.71 & $0.54,0.93$ & 0.01 \\
\hline Model 4\| & 1.00 & 0.81 & $0.63,1.05$ & 0.79 & $0.61,1.02$ & 0.74 & $0.57,0.96$ & 0.02 \\
\hline \multicolumn{9}{|l|}{ Vitamin E } \\
\hline \multicolumn{9}{|l|}{ Cases } \\
\hline$n$ & 156 & & & & & & & \\
\hline$\%$ & 3.2 & & & & & & & \\
\hline Model $1 \dagger$ & 1.00 & 0.79 & $0.62,1.01$ & 0.73 & $0.57,0.94$ & 0.71 & $0.56,0.92$ & 0.006 \\
\hline Model $2 \ddagger$ & 1.00 & 0.81 & $0.63,1.03$ & 0.75 & $0.58,0.96$ & 0.76 & $0.59,0.98$ & 0.02 \\
\hline Model $3 \S$ & 1.00 & 0.79 & $0.62,1.01$ & 0.73 & $0.56,0.94$ & 0.72 & $0.55,0.95$ & 0.02 \\
\hline Model $4 \|$ & 1.00 & 0.82 & $0.64,1.05$ & 0.77 & $0.59,0.99$ & 0.77 & $0.59,0.98$ & 0.04 \\
\hline \multicolumn{9}{|l|}{ Vitamin D } \\
\hline \multicolumn{9}{|l|}{ Cases } \\
\hline$n$ & 129 & & & & & & & \\
\hline$\%$ & $2 \cdot 7$ & & & & & & & \\
\hline Model 1† & 1.00 & 1.03 & $0.80,1.32$ & 1.01 & $0.78,1.29$ & 0.85 & $0.66,1.11$ & 0.20 \\
\hline Model $2 \ddagger$ & 1.00 & 1.04 & $0.81,1.33$ & 1.03 & $0.80,1.32$ & 0.87 & $0.67,1.13$ & 0.26 \\
\hline Model 3§ & 1.00 & 1.02 & $0.80,1.32$ & 1.00 & $0.77,1.29$ & 0.84 & $0.63,1.11$ & 0.19 \\
\hline Model 4\| & 1.00 & 1.09 & $0.83,1.41$ & 1.08 & $0.80,1.40$ & 0.94 & $0.69,1.27$ & 0.34 \\
\hline
\end{tabular}

* Median values of vitamins $A, K, E$ and $D$ intakes in each quartile were used to test for a linear trend across quartiles.

† Model 1 OR $(95 \% \mathrm{Cl})$ estimated by using logistic regression model adjusted for age and sex.

† Model 2 adjusted further for non-dietary factors including past history of hypertension, family history of diabetes, BMI, smoking status, hours of exercise, hours of walking and supplement use of vitamins $\mathrm{E}$ and $\mathrm{C}$ and multivitamins.

$\S$ Model 3 adjusted further for dietary factors including coffee, green tea and alcohol intakes and total energy, carbohydrate and Mg intakes (quartiles).

II Model 4 adjusted further mutually for dietary intakes of fat-soluble vitamins (quartiles). 
and supplemental consumptions of these vitamins on the risk of diabetes $^{(8)}$. Our findings indicated that high dietary intake of vitamin $\mathrm{E}$, but not vitamin $\mathrm{A}$, could benefit by reducing the risk of diabetes in our study subjects. The evidence from the scant previous literature showed inconsistent results; while dietary intake of both vitamin $\mathrm{E}$ (represented by $\alpha$-tocopherol) and vitamin A (represented by $\beta$-carotene) were inversely associated with the risk of diabetes in Swedish men ${ }^{(6)}$, neither dietary intake of most of the carotenoids in Dutch ${ }^{(7)}$ or Finnish $^{(8)}$, nor dietary intake of vitamin $\mathrm{E}$ in Chinese ${ }^{(9)}$ showed any association with the risk of diabetes. Moreover, while no association was found for dietary $\alpha$ - and $\beta$-carotenes, and only dietary $\beta$-cryptoxanthin was the most significant single carotenoid to inversely associate with the risk of diabetes in the Finnish study (relative risk $0.58,95 \%$ CI $0.44,0 \cdot 78)^{(8)}$, dietary consumptions of $\alpha$ - and $\beta$-carotenes (relative risk $0 \cdot 78,95 \%$ CI $0.64,0.95$ and $0.85,95 \%$ CI $0.70,1.03$, respectively), but not $\beta$-cryptoxanthin, were inversely associated with the diabetes risk in the Dutch study ${ }^{(7)}$. Going further for discrepancies, another Finnish study reported an increased risk of diabetes with high dietary vitamins A and E consumptions among male smokers; however, the association disappeared after multivariable adjustment ${ }^{(21)}$.

The exact mechanisms of why the reduced risk of diabetes is swinging within consumptions of dietary vitamins A and E in different populations are unclear. The sample size, participants' characteristics and diagnostic criteria of diabetes are hypothesised justifications ${ }^{(6-9,21)}$. For example, the Sweden study assessed the dietary intake by dietary records among older men aged $\geq 70$ years and diabetes status was retrieved from hospital registries ${ }^{(6)}$. The Finnish studies used FFQ for diet assessment among men and women aged $40-69$ years ${ }^{(8)}$ or male smokers aged 50-69 years ${ }^{(21)}$ and used the drug reimbursement register of the Finnish health insurance for diagnosing diabetes. The participants of the Chinese study aged 20-74 years and diabetes was diagnosed by an oral glucose tolerance test in some participants and by HbA1c levels in other participants ${ }^{(9)}$. In general, these results suggest other mechanisms besides the antioxidant effects of vitamins $\mathrm{A}$ and $\mathrm{E}$ are involved in reducing the risk of diabetes.

Ibarrola-Jurado et al. ${ }^{(10)}$ followed 1069 diabetes-free Spanish individuals at high risk of CVD, who had $\geq 3$ of the following risk factors: smoking, hypertension, dyslipidaemia, obesity or a family history of coronary artery disease for 5.5 years and reported that dietary consumption of vitamin $\mathrm{K}$ (phylloquinone) showed no association with fasting plasma glucose concentrations at baseline, but at the end of follow-up the risk of type 2 diabetes was reduced by $17 \%$ with each additional daily dietary intake of $100 \mu \mathrm{g}$ phylloquinone. Juanola-Falgarona et al. ${ }^{(22,23)}$ confirmed these observations in their cross-sectional and longitudinal assessments among Spanish population. Following 38094 Dutch men and women, aged 20-70 years for 10 years, yielded a multivariable hazard ratio for the risk of type 2 diabetes in the highest $v$. lowest quartiles of phylloquinone and menaquinones intake of 0.81 (95\% CI 0.66, 0.99) and 0.80 (95\% CI $0.62,1.02)$, respectively ${ }^{(11)}$. However, phylloquinone supplementation was not associated with the risk of diabetes in a systematic review that included studies investigating the relationship of intake and serum levels of vitamin $\mathrm{K}$ with risks of type 2 diabetes and CVD. However, this conclusion was based on the findings from only one clinical trial of vitamin $\mathrm{K}$ supplementation $^{(12)}$. So far, there is no clear biological mechanism for the inverse association between dietary vitamin $\mathrm{K}$ intake and the risk of type 2 diabetes observed in our study and previous studies $^{(10,11,22)}$. However, suggested pathways include the modulating effects of vitamin $\mathrm{K}$ on cytokines to decrease inflammation and improve insulin sensitivity ${ }^{(23)}$, and the vitamin-K-dependent biosynthesis of osteocalcin, a bonespecific protein that has regulating effects on both the expression of insulin genes ${ }^{(24)}$ and Ca metabolism ${ }^{(11)}$ that can reduce insulin resistance.

The last fat-soluble vitamin to be discussed (vitamin D) has gained a huge attention in the research area in the past decade $^{(5,13-16)}$. The rapidly increasing evidence postulates the impact of vitamin D on diabetes risk through its capacity to stimulate the expression of insulin receptors and to unite with the pancreatic $\beta$ cells' receptors enhancing insulin sensitivity ${ }^{(5)}$. Systematic reviews showed that some but not all clinical studies that examined the effect of 10-5000 $\mu \mathrm{g}$ vitamin D supplements' use for period of 2 months to 7 years have proven a modest favourable effect on impaired type 2 diabetes indicators such as fasting blood glucose and insulin sensitivity but not on glycated $\mathrm{Hb}^{(5,15,16)}$. However, the up-to-date studies that reported the association of dietary vitamin $\mathrm{D}$ and risk of type 2 diabetes showed inconsistent findings. Inverse associations were reported with the metabolic syndrome in the Women's Health Study ${ }^{(13)}$ but not with the risk of incident diabetes in the EPICInterAct study ${ }^{(14)}$. In our analysis, dietary vitamin $\mathrm{D}$ had no obvious association with the risk of type 2 diabetes.

We ran several sensitivity analyses to explore whether the reported association will be modified by varying levels of hypothesised effect modifiers; however, interactions of these factors with dietary vitamin $\mathrm{D}$ towards the risk of diabetes were not significant. Of a special interest was the effect modification of smoking status on the association between antioxidants and risk of diabetes. In fact, smoking not only increases the oxidative stress but also reduces serum tocopherols and carotenoid levels ${ }^{(25)}$. Kataja-Tuomola et $a l^{(21)}$ found no beneficial effects of dietary antioxidants consumption, including tocopherols and carotenoids, on the risk of diabetes in middle-aged male smokers. However, similar to our findings, there was no interaction with smoking status, which was proven in Dutch ${ }^{(7)}$ or Finnish studies ${ }^{(8)}$.

Our study, however, is not without limitations. The self-report of the outcome variable is one limitation; however, comparison with treatment and laboratory data showed very high sexspecific sensitivity and specificity for the self-reported physician-diagnosed diabetes in our participants ${ }^{(4,19)}$. Second, the dietary records-based validation study indicated that the FFQ underestimated participants' intake of fat-soluble vitamins by at least $20 \%{ }^{(19)}$. In this regard, we have to mention that the JACC FFQ did not include information about one important source of vitamin $\mathrm{K}$ in Japanese diet, natto (fermented soyabean). However, in general, the reproducibility of the FFQ was satisfactory and it was a good tool to sort individuals into the corresponding quartiles of dietary intake. Third, $64 \%$ of participants did not report their diabetes status 5 years from the baseline and 
accordingly our findings were based on data from $36 \%$ of eligible study participants. However, no significant differences in participants' characteristics such as age, BMI, dietary intakes of fat-soluble vitamins and other variables were found between individuals with and without missing data of diabetes history at 5 years from the baseline. Fourth, we do not have data on supplementation with fat-soluble vitamins, except for vitamin $\mathrm{E}$ which was reported by $2.6 \%$ of our study participants. Our baseline was in the late $80 \mathrm{~s}$, where Japanese population was unfamiliar with such supplementations; moreover, sensitivity analyses that excluded those supplement users did not alter the associations materially (data not shown). Last, we controlled for a wide range of hypothesised confounders; yet, the possibility of residual confounding remains.

\section{Conclusions}

Findings of this large Japanese prospective cohort study suggested that higher dietary intake of fat-soluble vitamins $\mathrm{K}$ and $\mathrm{E}$, but not A and D, were associated with lowered risk of type 2 diabetes among Japanese population. Public health implications of these findings draw the attention that dietary intake of these vitamins are accessible and affordable via stable diets of many populations, especially those who fear the use of chemical supplements. Therefore, dietary patterns that include different food sources abundant in vitamins $\mathrm{K}$ and $\mathrm{E}$, such as green leafy vegetables and fresh fish and meat, are suggested for the prevention of type 2 diabetes. However, future studies are needed to confirm these associations and to hypothesise plausible biological pathways for them.

\section{Acknowledgements}

The authors thank all staff members involved in this study for their valuable help in conducting the baseline survey and follow-up.

This work was supported by Grants-in-Aid for Scientific Research from the Ministry of Education, Science, Sports and Culture of Japan (MonbuKagaku-sho), Tokyo (nos 61010076, 2010074, 63010074, 1010068, 2151065, 3151064, 4151063, 5151069, 6279102, 11181101, 17015022, 18014011 and 16H06277) and by Grant-in-Aid from the Ministry of Health, Labour and Welfare, Health and Labor Sciences Research Grants, Japan (H23-Junkankitou [Seishuu]-Ippan-005, H26Junkankitou [Seisaku]-Ippan-001 and H29-Junkankitou [Seishuu]-Ippan-003).

The authors' contributions were as follows: H. I. and A. T. designed the research, conducted the analyses and prepared the manuscript; H. I., I. M. and A. T. made critical revisions of the manuscript; E. S. E. and H. I. had primary responsibility for final content. All authors read and approved the final manuscript

The authors declare that there are no conflicts of interest.

\section{Supplementary material}

For supplementary material/s referred to in this article, please visit https://doi.org/10.1017/S000711451800377X

\section{References}

1. International Diabetes Federation (2015) IDF Diabetes Atlas, 7th ed. Brussels, Belgium: International Diabetes Federation.

2. Hayama-Terada M, Muraki I, Imano H, et al. (2016) Diabetes trend and impact on risk of cardiovascular disease in middle-aged Japanese people - the CIRCS study. Circ J 80, 2343-2348.

3. Eshak ES, Iso H, Mizoue T, et al. (2013) Soft drink, $100 \%$ fruit juice, and vegetable juice intakes and risk of diabetes mellitus. Clin Nutr 32, 300-308.

4. Eshak ES, Iso H, Maruyama K, et al. (2018) Associations between dietary intakes of iron, copper and zinc with risk of type 2 diabetes mellitus: a large population-based prospective cohort study. Clin Nutr 37, 667-674.

5. Valdés-Ramos R, Guadarrama-López AL, Martínez-Carrillo BE, et al. (2015) Vitamins and type 2 diabetes mellitus. Endocr Metab Immune Disord Drug Targets 15, 54-63.

6. Arnlöv J, Zethelius B, Risérus U, et al. (2009) Serum and dietary beta-carotene and alpha-tocopherol and incidence of type 2 diabetes mellitus in a community-based study of Swedish men: report from the Uppsala Longitudinal Study of Adult Men (ULSAM) study. Diabetologia 52, 97-105.

7. Sluijs I, Cadier E, Beulens JW, et al. (2015) Dietary intake of carotenoids and risk of type 2 diabetes. Nutr Metab Cardiovasc Dis 25, 376-381.

8. Montonen J, Knekt P, Järvinen R, et al. (2004) Dietary antioxidant intake and risk of type 2 diabetes. Diabetes Care 27, 362-366.

9. Zhou C, Na L, Shan R, et al. (2016) Dietary vitamin C intake reduces the risk of type 2 diabetes in Chinese adults: HOMAIR and T-AOC as potential mediators. PLOS ONE 11, e0163571.

10. Ibarrola-Jurado N, Salas-Salvadó J, Martínez-González MA, et al. (2012) Dietary phylloquinone intake and risk of type 2 diabetes in elderly subjects at high risk of cardiovascular disease. Am J Clin Nutr 96, 1113-1118.

11. Beulens JW, van der A DL, Grobbee DE, et al. (2010) Dietary phylloquinone and menaquinones intakes and risk of type 2 diabetes. Diabetes Care 33, 1699-1705.

12. Rees K, Guraewal S, Wong YL, et al. (2010) Is vitamin K consumption associated with cardio-metabolic disorders? A systematic review. Maturitas 67, 121-128.

13. Liu S, Song Y, Ford ES, et al. (2005) Dietary calcium, vitamin $\mathrm{D}$, and the prevalence of metabolic syndrome in middle-aged and older US women. Diabetes Care 28, 2926-2932.

14. Abbas S, Linseisen J, Rohrmann S, et al. (2014) Dietary vitamin D intake and risk of type 2 diabetes in the European Prospective Investigation into Cancer and Nutrition: the EPIC-InterAct study. Eur J Clin Nutr 68, 196-202.

15. George PS, Pearson ER \& Witham MD (2012) Effect of vitamin D supplementation on glycaemic control and insulin resistance: a systematic review and meta-analysis. Diabet Med 29, e142-e150.

16. Poolsup N, Suksomboon N \& Plordplong N (2016) Effect of vitamin $\mathrm{D}$ supplementation on insulin resistance and glycaemic control in prediabetes: a systematic review and metaanalysis. Diabet Med 33, 290-299.

17. Tamakoshi A, Ozasa K, Fujino Y, et al. (2013) Cohort profile of the Japan Collaborative Cohort Study at final follow-up. J Epidemiol 23, 227-232.

18. Date C, Fukui M, Yamamoto A, et al. (2005) Reproducibility and validity of a self-administered food frequency questionnaire used in the JACC Study. J Epidemiol 15, S9-S23.

19. Eshak ES, Iso H, Yamagishi K, et al. (2018) Dietary intakes of fat soluble vitamins as predictors of mortality from heart failure in a large prospective cohort study. Nutrition $\mathbf{4 7}$, $50-55$. 
20. van Ballegooijen AJ, Cepelis A, Visser M, et al. (2017) Joint association of low vitamin D and vitamin $\mathrm{K}$ status with blood pressure and hypertension. Hypertension $\mathbf{6 9}$, $1165-1172$

21. Kataja-Tuomola MK, Kontto JP, Männistö S, et al. (2011) Intake of antioxidants and risk of type 2 diabetes in a cohort of male smokers. Eur J Clin Nutr 65, 590-597.

22. Juanola-Falgarona M, Salas-Salvadó J, Estruch R, et al. (2013) Association between dietary phylloquinone intake and peripheral metabolic risk markers related to insulin resistance and diabetes in elderly subjects at high cardiovascular risk. Cardiovasc Diabetol 12, 7.
23. Reddi K, Henderson B, Meghij S, et al. (1995) Interleukin 6 production by lipopolysaccharide-stimulated human fibroblasts is potently inhibited by naphthoquinone (vitamin K) compounds. Cytokine 7, 287-290.

24. Ferron M, Hinoi E, Karsenty G, et al. (2008) Osteocalcin differentially regulates $\beta$ cell and adipocyte gene expression and affects the development of metabolic diseases in wildtype mice. Proc Natl Acad Sci U S A 105, 5266-5270.

25. Gabriel HE, Liu Z, Crott JW, et al. (2006) A comparison of carotenoids, retinoids, and tocopherols in the serum and buccal mucosa of chronic cigarette smokers versus nonsmokers. Cancer Epidemiol Biomarkers Prev 15, 993-999. 